\title{
Public health response to two incidents of confirmed MERS-CoV cases travelling on flights through London Heathrow Airport in 2014 - lessons learnt
}

F Parry-Ford (frances.parryford@phe.gov.uk) ${ }^{1}$, N Boddington ${ }^{1}$, R Pebody $^{1}$, N Phin ${ }^{1}$, on behalf of the Incident Management Team ${ }^{2}$

1. Centre for Infectious Disease Surveillance and Control, Public Health England, London, United Kingdom

2. Members of the team are listed at the end of the article

Parry-Ford F, Boddington N, Pebody R, Phin N, on behalf of the Incident Management Team. Public health response to two incidents of confirmed MERS-CoV cases travelling on flights through London Heathrow Airport in 2014 - lessons learnt. Euro Surveill, 2015;20(18):pii=21114. Available online: http://www. eurosurveillance.org/ViewArticle.aspx?Articleld=21114

In May 2014, Public Health England was alerted to two separate laboratory-confirmed cases of Middle East respiratory syndrome coronavirus (MERS-CoV) infection who transited through London Heathrow Airport while symptomatic on flights from Saudi Arabia to the United States of America. We present the rationale for the public health response to both incidents, and report results of contact tracing. Following a risk assessment, passengers seated two seats around the cases were prioritised for contact tracing and a proactive media approach was used to alert all passengers on the planes of their possible exposure in both incidents. In total, 64 United Kingdom (UK) residents were successfully contacted, 14 of whom were sat in the priority area two seats all around the case(s). Five passengers reported respiratory symptoms within 14 days of the flight, but all tested were negative for MERSCoV. Details of non-UK residents were passed on to relevant World Health Organization International Health Regulation focal points for follow-up, and no further cases were reported back. Different approaches were used to manage contact tracing for each flight due to variations in the quality and timeliness of the passenger contact information provided by the airlines involved. No evidence of symptomatic onward transmission was found.

\section{Introduction}

On 2 May 2014, Public Health England (PHE) was alerted by the public health authorities in the United States (US) of a laboratory-confirmed case of Middle East respiratory syndrome coronavirus (MERS-CoV) infection who had transited through London Heathrow Airport, United Kingdom (UK) on a flight from Saudi Arabia to the US [1]. Nine days later, the US authorities notified PHE of a second, unlinked, laboratoryconfirmed MERS-CoV case that had also travelled from Saudi Arabia to the US and transited through Heathrow Airport [1].
This article describes the contact tracing approaches undertaken for both in-bound flights, compares the issues and outcomes and provides recommendations on future approaches. The results of contact tracing of UK nationals on the same onward flights from London to the US as the cases are also reported.

MERS-CoV is a novel coronavirus, first detected in a patient in 2012 [2]. As of 16 April 2015, the World Health Organization (WHO) have been notified of 1,106 cases of MERS-CoV, with 421 deaths [3]. The vast majority of cases have occurred in Saudi Arabia and the United Arab Emirates. However, exported MERS-CoV cases have occurred in several countries outside the Middle East the majority travelling by air [4]. The public health response to many of these cases involved follow up of close contacts on planes to detect any evidence of onward transmission. Only limited secondary transmission has been demonstrated to date around such exported cases [5].

Two earlier imported laboratory-confirmed MERS-CoV cases from the Middle East have been documented in the UK. Of these, the first was transported to the UK via private air ambulance and the second travelled on a commercial flight. Both were symptomatic during their flights. No secondary cases were identified among the contacts on either of these flights, however two family contacts of the second case were later identified as confirmed cases following transmission in the UK in household and hospital settings $[6,7]$.

Outside of these incidents, we are aware of four further published reports of confirmed MERS-CoV cases travelling on commercial flights, including two cases who were reported to have travelled while symptomatic to Greece and Italy, respectively [8,9]. Another was an asymptomatic case who travelled to Malaysia and later developed symptoms upon arrival [10]. In May 2014, two laboratory-confirmed cases travelled on the 
same flight from Saudi Arabia to the Netherlands while symptomatic [11]. Variable approaches were used for the contact tracing of flight contacts in each of these incidents, however, all failed to identify any evidence of onward transmission to other passengers.

London Heathrow Airport is a key air transport hub, and more passengers from the Middle East travel to, or transit through the UK than any other country in Europe [12]. This paper describes the public health response to two further laboratory-confirmed MERS-CoV cases on commercial flights, provides further evidence on the potential transmissibility of MERS-CoV during air travel, and informs the public health response to possible future incidents. The paper also explores alternative options to identify possible cases by using the media and public information messages to raise awareness among those travelling to and returning from the Middle East, particularly in circumstances when passenger lists may not be available.

\section{Incident 1 - Flight Riyadh to London}

Heathrow, 24 April 2014 (with onward travel to Chicago)

On Friday 2 May 2014, PHE was alerted by the US Centers for Disease Control and Prevention (CDC) of a reported probable case of MERS-CoV infection, positive on screening by PCR but awaiting confirmation by the national reference centre. The case had travelled eight days earlier from Riyadh, Saudi Arabia to Chicago, US, on 24 April, transiting through London Heathrow Airport. Confirmation was received from CDC at 8p.m. GMT on 2 May 2014 [1]. The case was a healthcare worker who had been working in a Saudi Arabian healthcare institution that treated MERS-CoV patients [1]. The case first felt unwell on 18 April. Six days later, they were symptomatic with fever and myalgia, but without respiratory symptoms, during the seven hour flight from Riyadh to London. They spent approximately two hours at London Heathrow before boarding a flight to Chicago. Following the initial notification on 2 May, PHE immediately convened an incident management team.

\section{Considerations for contact tracing}

Although the risk of onward transmission to passengers and crew on board the flight was considered to be very low, given the recent emergence of MERS-CoV it was agreed with the CDC to adopt a precautionary approach and undertake contact tracing on the basis of protecting public health, as well as to add to the existing evidence base for risk of transmission on aircraft and inform future responses.

In the UK it was agreed that passengers in the immediate vicinity of the case were a priority and should be contacted and followed up. At the time of the incident no specific international guidance on contact tracing on aircraft for MERS-CoV cases existed, therefore guidance on severe acute respiratory syndrome (SARS) was considered as the most analogous and suitable for our investigation. The current European Centre for Disease Prevention and Control (ECDC) Risk Assessment Guidelines for Infectious Diseases transmitted on Aircraft (RAGIDA) suggests that for SARS, contact tracing should be comprehensive, and may extend to passengers up to seven rows around the case, or even the entire plane, whereas WHO guidance suggests two rows in front and behind the case and the row they are sat in [13,14]. Given that there has been little evidence from previous incidents to suggest MERS-CoV is easily transmissible on aircrafts, it was decided to adopt a more pragmatic approach based on the WHO guidance.

Passengers seated in the two seats all around the case were deemed to be 'priority' contacts. The remaining passengers and crew were to be alerted to the situation and advised on the actions to take should they develop respiratory symptoms within the 14 days following the flight.

The flight manifest and contact details of the passengers were requested from the airline. Previous experiences of obtaining passenger details for contact tracing suggested this information could take some time to obtain. A proactive media approach was developed to rapidly initiate the contact tracing process, on the basis that the forthcoming weekend of 3 to 5 May included a public holiday, and that eight days of the 14-day incubation period had already passed. The press release was timed to coincide with the announcement of confirmatory test results from the US and was issued on Friday 2 May with details of the flight number, and advice to any passengers with respiratory symptoms on that flight to seek medical advice through NHS 111, (a UK telephone service for health advice provided by the National Health Service), due to limited primary care access over the weekend. An algorithm was developed to support call handlers to manage calls from members of the public who had been on the flight.

\section{Follow-up and criteria for testing}

The passenger contact details were provided by the airline within hours of request and contained names, nationalities and contact details for all passengers. Priority contacts who were identified as being resident in the UK from their contact details, were initially interviewed centrally by staff at the Centre for Infectious Disease Surveillance and Control (CIDSC) on the evening of Friday 2 May, and their details were passed to their local health protection team for follow-up. They were informed of a possible exposure to a confirmed MERS-CoV case and asked whether they had developed any respiratory symptoms since the flight.

The definition of a possible case, and criteria for testing, was anyone who was on the flight and who had experienced respiratory symptoms, within 14 days of the flight. This definition is synonymous to the PHE close contact definition for MERS-CoV [15]. Symptomatic contacts were assessed by a health professional over the phone, and were asked to self-isolate or attend 


\section{FIGURE 1}

Algorithm for management of flight contacts in two incidents of confirmed MERS-CoV cases travelling on flights through London Heathrow Airport, United Kingdom, May 2014

\begin{tabular}{|c|c|c|}
\hline \multicolumn{3}{|c|}{$\begin{array}{l}\text { Advise contact of possible exposure to MERS-CoV } \\
\text { Experienced any respiratory symptoms or fever in the } 14 \text { days since } \\
\text { the flight? }\end{array}$} \\
\hline Yes & \multirow[b]{4}{*}{ ment } & No \\
\hline Recovered & & \multirow{3}{*}{$\begin{array}{l}\text { - Explain symptoms } \\
\text { - Advise to contact PHE if they } \\
\text { develop symptoms within } 14 \text { days of } \\
\text { flight }\end{array}$} \\
\hline$\nabla^{\text {No }}$ & & \\
\hline Clinical assessment & & \\
\hline Mild & Severe & \multirow{2}{*}{$\begin{array}{l}\text { Follow-up after } 14 \text { days if sat in } \\
\text { priority area }\end{array}$} \\
\hline & & \\
\hline isolation & admission & \\
\hline \multicolumn{2}{|r|}{ 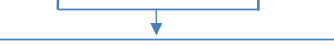 } & \\
\hline \multicolumn{2}{|c|}{$\begin{array}{l}\text { MERS-CoV testing requested } \\
\text { - Nose and throat swabs } \\
\text { - Sputum }\end{array}$} & \\
\hline
\end{tabular}

MERS-CoV: Middle East respiratory syndrome coronavirus; PHE: Public Health England.

hospital if warranted. Lower respiratory samples and/ or nose and throat swabs were arranged using appropriate infection control precautions [16]. Asymptomatic contacts were asked to report any respiratory symptoms or fever that developed within the remaining 14 day period from the flight. All priority contacts were followed-up by local health protection teams (Figure 1) for at least 14 days since their exposure - the maximum putative incubation period of MERS-CoV.

The details of all passengers that were non-UK residents were sent to relevant national focal points via the bilateral International Health Regulations (IHR) and European Early Warning Response System (EWRS) over the public holiday weekend, and feedback on the health status of these contacts was requested.

In addition to passengers on the Riyadh to London flight, details for those on the London to Chicago flight who had returned to the UK were sent to PHE by the CDC and were prioritised and followed up as above.

Following the weekend press release, all remaining passengers resident in the UK on the flight were actively contacted on Tuesday, 6 May, following the public holiday weekend. Where the address of passengers was known, their contact details were passed on to the relevant local health protection team to make contact. Otherwise passengers were informed centrally by CIDSC who gathered the relevant contact details. Passengers were asked to report whether they had experienced any respiratory symptoms within 14 days of the flight. If yes, they were managed in the same way as the priority contacts, but they were not followed-up after 14 days.

\section{Incident 2 - Flight Jeddah to London Heathrow, 1 May 2014 (with onward travel to Boston)}

On 11 May 2014, PHE was alerted to a second probable case of MERS-CoV infection (later confirmed) who had travelled from Jeddah, Saudi Arabia to Boston, US via London Heathrow Airport 10 days previously, on 1 May 2014 [1]. The case became symptomatic on the day of the flight and was symptomatic on board the six hour UK bound flight with symptoms of fever, chills, myalgia and a slight cough.

\section{Contact tracing}

A basic passenger manifest containing names and seat numbers of all passengers on the flight was available from the airline within 24 hours of the notification from the CDC. The passenger contact details were only provided on 14 May, at the end of the 14 day follow-up period.

In a similar fashion to incident 1 , a pro-active press release was issued on 12 May to alert passengers, and the NHS 111 algorithm was adapted to include passengers on this flight. Given that the maximum incubation period of 14 days was exceeded by the time information was available, only the 17 passengers who sat in the immediate two adjacent seats to the case were identified for active contact tracing, however contact details were not available for seven of them. They were asked about any respiratory symptoms that might have developed within 14 days of the flight. If they had developed symptoms within 14 days of the flight, and were still symptomatic, they were clinically assessed, asked to self-isolate and sampling was undertaken as described above.

Where available, the details of non-UK residents sitting in the two seats all around the case were passed to the respective national focal point via bilateral IHR and EWRS mechanisms. The CDC provided details on passengers who were on the flight from London to Boston, but had returned to the UK, and they were contacted if they were seated within two seats all around the case.

\section{Contact tracing results}

Results of the contact tracing are depicted in Figure 2.

Incident 1 - Flight Riyadh to London Heathrow There were 178 passengers on the flight manifest on 24 April 2014, including the case. Of these, 49 passengers were UK residents of whom four were considered priority contacts as they were seated within two seats all around the case; the remaining 45 were seated elsewhere in the plane. PHE successfully contacted 36 of these passengers. None of the four priority passengers reported acute respiratory symptoms in the 14 days since the flight; however, three of the non-priority passengers reported mild respiratory symptoms during 
FIGURE 2

Results of contact tracing for UK residents in two incidents of confirmed MERS-CoV cases travelling on flights through London Heathrow Airport, UK, May 2014

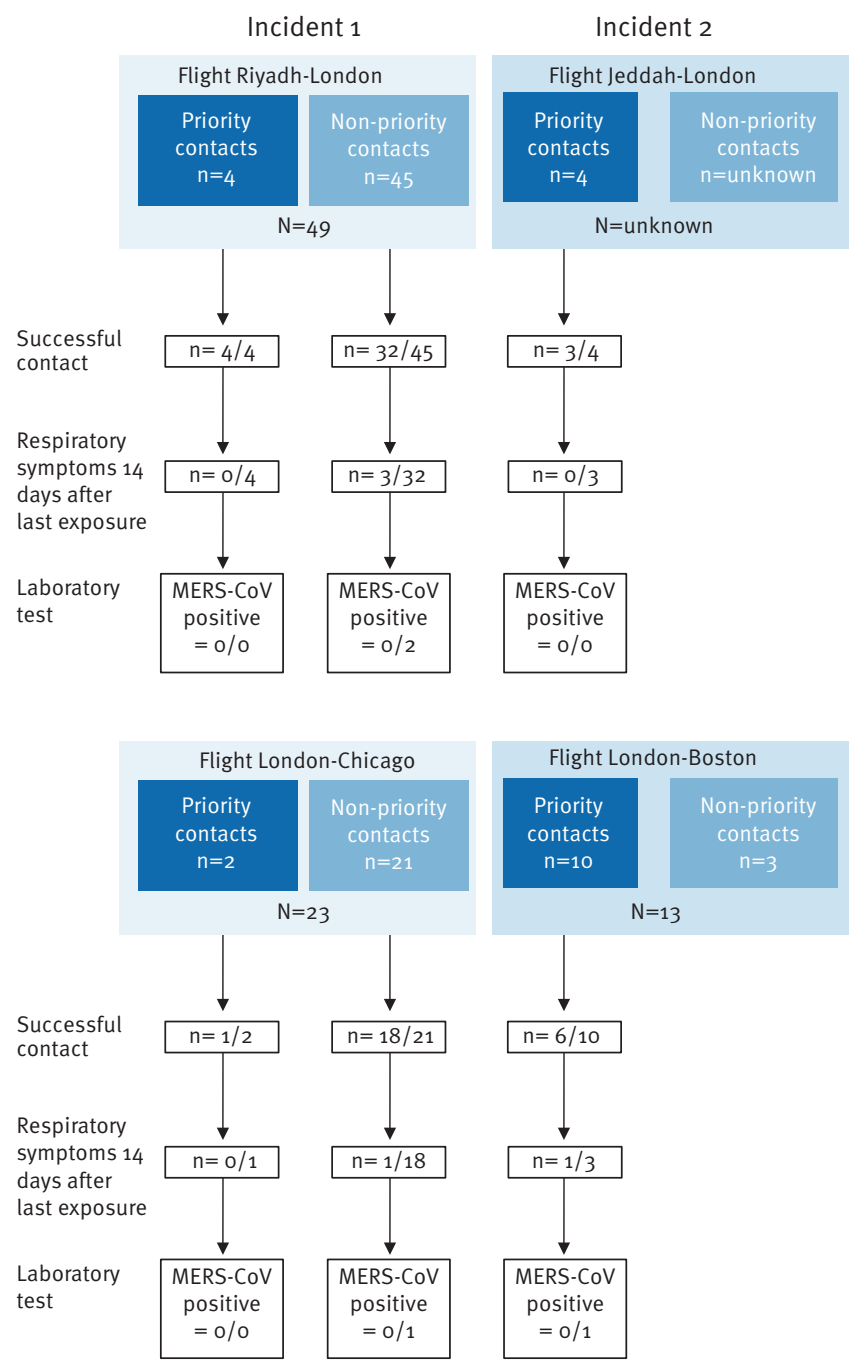

MERS-CoV: Middle East respiratory syndrome coronavirus; UK: United Kingdom.

Information on contact tracing of international contacts on the flights is not displayed in this figure.

this period and were still symptomatic. None required hospitalisation.

\section{Testing of symptomatic contacts}

The two symptomatic contacts were sampled and tested. Both were negative for MERS-CoV by PCR testing. One contact was positive for rhinovirus and the other one was positive for influenza B virus. The third symptomatic contact was not tested as they did not agree to be further contacted by the local health protection team.

\section{Non-United Kingdom residents}

Of the 128 passengers who were non-UK residents or nationals, 13 were seated within two seats all around the case, the remainder were seated elsewhere on the plane. Details of these passengers were passed to respective international counterparts for contact tracing. Of these 13 passengers that were non-UK residents and were seated in the priority area, seven were contacted and all were asymptomatic. There was no feedback received about the non-UK passengers seated elsewhere on the plane.

Incident 2 - Flight Jeddah to London Heathrow The flight manifest identified 17 passengers on the flight on 1 May 2014 who were priority contacts. Four of these passengers were UK residents and six were international contacts. No contact information was available for seven of the passengers. Of the four UK contacts, three were successfully contacted, none of whom reported any acute respiratory symptoms within 14 days of the flight. No further information was received on the international passengers.

\section{Onward flights from London Heathrow to the United States}

The CDC informed PHE of contacts on the onward flights from London to the US.

\section{Incident 1}

The case travelled to Chicago on 24 April and 23 UK contacts were identified on this flight, two of these were considered priority contacts. Contact was made with one who did not report any respiratory symptoms within nine days from the flight. The other 21 UK residents on the flight were considered non-priority contacts, and 18 were successfully contacted, of whom one was symptomatic within the 14 days after the flight. Upper respiratory tract samples tested negative for MERS-CoV and for other respiratory viruses.

\section{Incident 2}

The case travelled to Boston on 1 May and 13 UK contacts were identified on this flight. Ten of these contacts were considered priority contacts, of which six were successfully contacted and one was found to be symptomatic. This contact was found to be positive for streptococcal A infection, but negative for MERS$\mathrm{CoV}$ and a respiratory virus panel (adenovirus, respiratory syncytial virus, parainfluenza, rhinovirus, human metapneumovirus and influenza $A$ and $B$ virus).

\section{Discussion and conclusions}

Given the prominence of the UK, and particularly London Heathrow, as a key air transport hub connected with the Middle East and continued reporting of new MERS-CoV cases from that region, it is probable that similar incidents will occur in the future. This may happen with higher frequency during times of increased local transmission in the Middle East as was observed in April-May 2014. Although the incidence of MERS$\mathrm{CoV}$ has remained low compared with this particular peak period, a smaller peak of cases occurred in Saudi Arabia during early spring $\mathbf{2 0 1 5}$, suggesting there may 
yet be an aspect of local seasonality to MERS-CoV transmission [17]. Contact tracing investigations, such as the one described, have significant resource implications for public health due to the effort required to identify and follow up passengers on a flight.

In total, there were 89 UK contacts on board these flights, of which 20 were priority contacts and 69 non-priority contacts. The contact tracing carried out for the two flights to the UK described in detail in this paper and among UK residents on the two flights to the US, did not identify any further symptomatic cases of MERS-CoV infection. This adds to the growing body of evidence of the lack of transmission to date from symptomatic MERS-CoV cases during air travel [8-11].

During the investigation we only took clinical samples from symptomatic contacts. This is a limitation, as MERS-CoV infection has been observed to cause mild disease and asymptomatic infections which we may not have identified by the approach outlined in this paper [6]. However, we used a low threshold for symptoms requiring testing to increase the likelihood of identifying mild cases. We also did not test contacts serologically unless there was an indication of secondary transmission from initial tests, as serology was not considered to be proportionate to the public health risk based on evidence from other investigations. We were unfortunately unable to contact all the people considered to be priority contacts, which is a further limitation of our investigation. However, no cases of MERS-CoV infection have been confirmed in the UK since the flights. One priority contact reported symptoms, however they did not accept further contact with the local health protection team, and would not attend for testing. They were provided with advice on the symptoms, and how to contact health services should their condition deteriorate.

The two incidents illustrate the possible challenges and unpredictability of public health investigations. In both, public health authorities only became aware of the incidents sometime after the exposure. In the first incident a full flight manifest with contact details for most of the passengers, was available within 12 hours of notification, enabling an effective, although resource intensive, response. In the second incident the contact details of passengers were not available until several days following the first notification. Moreover, the details, when available, were incomplete, and contact tracing did not take place until after the end of the maximum putative incubation period of 14 days, minimising the potential public health benefits of the investigation. We did not receive information on the health status of all the non-UK contacts that were passed to other national focal points. This is a further challenge in the investigation of these incidents, as the majority of flight contacts were non-UK nationals.

The difficulties of undertaking rapid and effective contact tracing on flights due to the variability of the information made available by the respective airlines, and the speed at which it is received, even if considerable effort and resources are committed is highlighted here. The public health responsibilities of conveyancers are outlined in the International Health Regulations [18]. There are specific regulations regarding the provision of passenger contact information when an incident of public health concern is identified during the flight. However, there is no public health legislation requiring conveyancers to retain information such as passenger manifests or contact details for contact tracing in the event that disease is identified after the flight. Although both airlines in these described incidents were very co-operative, it is recommended that the ability to rapidly access plane manifests with adequate information to enable public health investigation be further strengthened. Declaring MERS-CoV infection as a notifiable disease within the UK may contribute to this; however, administrative practices among airlines with regards to the length of time flight manifests and contact details are retained would also need to be addressed. New UK legislation (in development) is likely to mandate that best efforts are made to provide passenger contact information for such public health investigations, however, currently such airline co-operation is on a voluntary basis [19].

Due to past difficulties in obtaining passenger information, PHE sought to explore other mechanisms to alert passengers on these flights in a timely manner. In both incidents a pro-active media approach linked to a telephone help-line service was applied in an attempt to alert passengers in the UK prior to the passenger information becoming available. In practice no passenger contacts were identified through this mechanism that we are aware of. Although not formally measured, the incident response highlighted a lack of awareness of MERS-CoV infections among passengers travelling to and from the Middle East. Many of the passengers spoken to during the contact tracing expressed very limited knowledge of MERS-CoV symptoms or risks. Following the incidents, as a more general measure PHE adapted $C D C$ information materials for display in airports in order to raise awareness of MERS-CoV among the general public and provide advice on the symptoms and how to access medical attention. These have also been circulated to airlines with direct flights to the Middle East; however, uptake and use has been limited due to airports expressing commercial concerns. Finally, the option of alerting all non-priority contacts via text message was considered, and although this was not done during the incidents, it could be a resource-effective approach as a phone number was the most consistently available contact information within the manifest.

The response to this incident required significant resources at both a national and local level, as well as international collaboration. Over the course of the weekend and the initial incident response, several incident meetings were held, with around 20 senior staff in attendance across epidemiology and surveillance, 
port health, microbiology services, operational health protection staff, communications and the NHS. Within PHE four scientists, as well as seven specialist registrars worked full time on the contact tracing and incident response over several days. At a local level, 18 local health protection teams were involved in the contact tracing and follow-up. This sort of contact tracing requires significant person-time, and for this reason, any decision to deploy this level of resource should be taken judiciously, particularly in the emerging evidence of limited transmissibility in such settings.

Supranational organisations such as WHO and ECDC and the established information processes such as IHR national focal points, and the EWRS play a key role in incidents such as this. On both flights, the majority of persons were non-UK residents, and using these systems we were able to pass on details, and receive information on the health status of non-UK contacts with international counterparts. However, not all focal points responded, and so there were still gaps in our information. Given the international nature of air travel, an enhanced role of ECDC or WHO could be to encourage countries to respond to requests for information on identified contacts, providing more reliable information on transmission in the early stages of an emerging infection.

There are still some gaps in understanding of the risk of transmission of MERS-CoV from patients who are asymptomatic, or who do not have respiratory symptoms $[20,21]$ There is evidence of transmission from cases with mild symptoms or who are asymptomatic [22]. However, documented episodes of human-tohuman transmission have been concentrated in healthcare settings, or within households. This suggests that close and prolonged contact with the case is required [23]. The first case reported here had no respiratory symptoms during the flight and may have posed a very low transmission risk if MERS-CoV transmissibility is similar to other respiratory viruses. The second case had a slight cough, and so may have represented a slightly greater infection risk through the respiratory route. $A$ better understanding of the risk of transmissibility will allow the scale of the public health response to be modified. However, until a better understanding is achieved, the public health response to such events needs to remain precautionary. In the meantime, the need to develop less resource-intensive methods of contact follow-up and investigation persists.

In light of the UK experience, and the limited evidence of in-flight transmission of MERS-CoV from other investigations, we would argue that contact tracing and follow-up of the entire plane, or up to the maximum range of seven rows either side of the case (as per the RAGIDA guidelines for SARS) is not a proportionate response for cases of MERS-CoV travelling on flights in general. We recommend that based on current evidence, contact tracing of two rows in front and behind, or as in our investigation, two seats all around the case, represents a more appropriate approach in most circumstances, although this needs to be evaluated on a case-by-case basis.

Members of the Incident Management Team

Joanne Freedman, Gavin Dabrera, Hongxin Zhao, Mathibalasingham Chandrakumar, GeeYen Shin, Richard Holliman, Darren Ready, Mel Sirotkin, Diana Grice, Maria Zambon, Mike Catchpole.

Acknowledgments

All members of the PHE incident management team, those who contributed to the response and provided comments on the article, including local PHE Health Protection Teams and colleagues from NHS England.

\section{Conflict of interest}

None declared.

Authors' contributions

F Parry-Ford and N Boddington agreed the initial outline for the article and wrote the first draft. All authors made a substantive contribution to the writing of the final manuscript.

References

1. Bialek SR, Allen D, Alvarado-Ramy F, Arthur R, Balajee A, Bell D, et al. First confirmed cases of Middle East respiratory syndrome coronavirus (MERS-CoV) infection in the United States, updated information on the epidemiology of MERS-CoV infection, and guidance for the public, clinicians, and public health authorities - May 2014. MMWR Morb Mortal Wkly Rep. 2014;63(19):431-6. PMID:24827411

2. Corman VM, Eckerle I, Bleicker T, Zaki A, Landt O, Eschbach Bludau M, et al. Detection of a novel human coronavirus by real-time reverse-transcription polymerase chain reaction. Euro Surveill. 2012;17(39):20285. PMID:23041020

3. World Health Organization (WHO). Disease Outbreak News. Middle East Respiratory Syndrome coronavirus (MERS-CoV) - Saudi Arabia. 16 Apr 2015. Geneva: WHO. Available from: http://www.who.int/csr/don/16-april-2015-mers-saudi-arabia/ en/

4. European Centre for Disease Prevention and Control (ECDC). Updated rapid risk assessment. Severe respiratory disease associated with Middle East respiratory syndrome coronavirus (MERS-CoV) Fifteenth update. Stockholm: ECDC. 8 Mar 2015. Available from: http://ecdc.europa.eu/en/publications/ Publications/MERS_update_08-Mar2014.pdf

5. World Health Organization (WHO). Middle East respiratory syndrome coronavirus (MERS-CoV): Summary of Current Situation, Literature Update and Risk Assessment-as of 5 February 2015. Geneva: WHO. 5 Feb 2015. Available from: http://www.who.int/csr/disease/coronavirus_infections/mers5-february-2015.pdf

6. Bermingham A, Chand MA, Brown CS, Aarons E, Tong C, Langrish C, et al. Severe respiratory illness caused by a novel coronavirus, in a patient transferred to the United Kingdom from the Middle East, September 2012. Euro Surveill. 2012;17(40):20290. PMID:23078800

7. Health Protection Agency (HPA) UK Novel Coronavirus Investigation team. Evidence of person-to-person transmission within a family cluster of novel coronavirus infections, United Kingdom, February 2013. Euro Surveill. 2013;18(11):20427. PMID:23517868

8. Tsiodras S, Baka A, Mentis A, Iliopoulos D, Dedoukou X, Papamavrou G, et al. A case of imported Middle East Respiratory Syndrome coronavirus infection and public health response, Greece, April 2014. Euro Surveill. 2014;19(16):20782. http://dx.doi.org/10.2807/1560-7917.ES2014.19.16.20782 PMID:24786258 
9. Puzelli S, Azzi A, Santini MG, Di Martino A, Facchini M, Castrucci MR, et al. Investigation of an imported case of Middle East Respiratory Syndrome Coronavirus (MERS-CoV) infection in Florence, Italy, May to June 2013. Euro Surveill. 2013;18(34):20564. http://dx.doi.org/10.2807/1560-7917. ES2013.18.34.20564 PMID:23987829

10. Premila Devi J, Noraini W, Norhayati R, Chee Kheong C, Badrul AS, Zainah S, et al. Laboratory-confirmed case of Middle East respiratory syndrome coronavirus (MERS-CoV) infection in Malaysia: preparedness and response, April 2014. Euro Surveill. 2014;19(18):20797. http://dx.doi.org/10.2807/15607917.ES2014.19.18.20797 PMID:24832116

11. Kraaij-Dirkzwager M, Timen A, Dirksen K, Gelinck L, Leyten $E$, Groeneveld P, et al. Middle East respiratory syndrome coronavirus (MERS-CoV) infections in two returning travellers in the Netherlands, May 2014. Euro Surveill. 2014;19(21):20817. http://dx.doi.org/10.2807/1560-7917.ES2014.19.21.20817 PMID:24906375

12. European Centre for Disease Prevention and Control (ECDC). Updated rapid risk assessment: Severe respiratory disease associated with Middle East respiratory syndrome coronavirus (MERS-CoV). Tenth update. Stockholm: ECDC. 31 May 2014. Available from: http://www.ecdc.europa.eu/en/publications/ Publications/RRA-Middle-East-respiratory-syndromecoronavirus-update10.pdf

13. European Centre for Disease Prevention and Control (ECDC). Technical report. Risk assessment guidelines for infectious diseases transmitted on aircraft. Stockholm: ECDC. Jun 2009. Available from: http://www.ecdc.europa.eu/en/publications/ publications/0906 ter risk assessment guidelines_for infectious diseases transmitted on aircraft.pdf

14. World Health Organization (WHO). WHO recommended measures for persons undertaking international travel from areas affected by severe acute respiratory syndrome (SARS). Wkly Epidemiol Rec. 2003;78(14):97-9. PMID:12723281

15. Public Health England (PHE). Investigation and public health management of close contacts of Middle East respiratory syndrome coronavirus (MERS-CoV) cases. London: PHE. 16 May 2014. Available from: https://www.gov.uk/government/ uploads/system/uploads/attachment_data/file/360918/ Algorithm_contact_v16.pdf

16. Public Health England (PHE). MERS-CoV Infection control guidance for possible or confirmed cases. London: PHE. 28 Jun 2013. Available from: https://www.gov.uk/government/ uploads/system/uploads/attachment_data/file/361569/MERSCoV_infection_control.pdf

17. World Health Organization (WHO) Eastern Mediterranean Regional Office. (EMRO). MERS-CoV Situation Update. Cairo: WHO EMRO. 31 Mar 2015. Available from: http://www. emro.who.int/images/stories/csr/documents/MERS-CoV_ March 2015.pdf

18. World Health Organization (WHO). International Health Regulations. 2nd Edition. Geneva: WHO. 2005. Available from: http://whqlibdoc.who.int/publications/2008/9789241580410_ eng.pdf?ua=1

19. Department of Health. Health protection (ships and aircraft) regulations: A consultation. London: Department of Health. 2013. Available from: https://www.gov.uk/government/ uploads/system/uploads/attachment_data/file/225162/ Health_protection_ships_and_aircrafts_regulations consultation_document.pdf

20. The WHO Mers-Cov Research Group. State of Knowledge and Data Gaps of Middle East Respiratory Syndrome Coronavirus (MERS-CoV) in Humans. PLoS Curr. 2013;5.

21. de Sousa R, Reusken C, Koopmans M. MERS coronavirus: data gaps for laboratory preparedness. J Clin Virol. 2014;59(1):4-11. http://dx.doi.org/10.1016/j.jcv.2013.10.030 PMID:24286807

22. Omrani AS, Matin MA, Haddad Q, Al-Nakhli D, Memish ZA, Albarrak AM. A family cluster of Middle East Respiratory Syndrome Coronavirus infections related to a likely unrecognized asymptomatic or mild case. Int J Infect Dis. 2013;17(9):e668-72. http://dx.doi.org/10.1016/j. ijid.2013.07.001 PMID:23916548

23. Al-Tawfiq JA, Memish ZA. Middle East respiratory syndrome coronavirus: transmission and phylogenetic evolution. Trends Microbiol. 2014;22(10):573-9. http://dx.doi.org/10.1016/j. tim.2014.08.001 PMID:25178651 